\title{
CAPTURE OF TROJANS BY JUMPING JUPITER
}

\author{
David NesvornÝ ${ }^{1}$, David VokrouhlickÝ ${ }^{2}$, AND Alessandro Morbidelli ${ }^{3}$ \\ ${ }^{1}$ Department of Space Studies, Southwest Research Institute, 1050 Walnut St., Suite 300, Boulder, CO 80302, USA \\ ${ }^{2}$ Institute of Astronomy, Charles University, V Holešovičkách 2, 18000 Prague 8, Czech Republic \\ ${ }^{3}$ Département Cassiopée, University of Nice, CNRS, Observatoire de la Côte d'Azur, Nice, F-06304, France \\ Received 2012 December 21; accepted 2013 March 11; published 2013 April 12
}

\begin{abstract}
Jupiter Trojans are thought to be survivors of a much larger population of planetesimals that existed in the planetary region when planets formed. They can provide important constraints on the mass and properties of the planetesimal disk, and its dispersal during planet migration. Here, we tested a possibility that the Trojans were captured during the early dynamical instability among the outer planets (aka the Nice model), when the semimajor axis of Jupiter was changing as a result of scattering encounters with an ice giant. The capture occurs in this model when Jupiter's orbit and its Lagrange points become radially displaced in a scattering event and fall into a region populated by planetesimals (that previously evolved from their natal transplanetary disk to $\sim 5$ AU during the instability). Our numerical simulations of the new capture model, hereafter jump capture, satisfactorily reproduce the orbital distribution of the Trojans and their total mass. The jump capture is potentially capable of explaining the observed asymmetry in the number of leading and trailing Trojans. We find that the capture probability is $(6-8) \times 10^{-7}$ for each particle in the original transplanetary disk, implying that the disk contained (3-4) $\times 10^{7}$ planetesimals with absolute magnitude $H<9$ (corresponding to diameter $D=80 \mathrm{~km}$ for a $7 \%$ albedo). The disk mass inferred from this work, $M_{\text {disk }} \sim 14-28 M_{\text {Earth }}$, is consistent with the mass deduced from recent dynamical simulations of the planetary instability.
\end{abstract}

Key words: Kuiper belt: general - minor planets, asteroids: general - planets and satellites: dynamical evolution and stability - planets and satellites: formation

Online-only material: color figures

\section{INTRODUCTION}

Jupiter Trojans are populations of small bodies with orbits similar to that of Jupiter. They clump around two equilibrium points of the three-body problem, known as the Lagrange $L_{4}$ and $L_{5}$ points, with semimajor axes $a \simeq a_{\text {Jup }}$, eccentricities $e \lesssim 0.15$, inclinations $i \lesssim 35^{\circ}$, and $\Delta \lambda=\lambda-\lambda_{\text {Jup }} \sim \pm 60^{\circ}$, where $\lambda$ is the mean longitude and index "Jup" denotes Jupiter. The angle $\Delta \lambda$ oscillates with a period of $\sim 150 \mathrm{yr}$ and full libration amplitude up to $D \simeq 70^{\circ}$ (see Marzari et al. 2002 for a review). The total population of Jupiter Trojans is estimated to be $\gtrsim 10 \%$ of the main asteroid belt (e.g., Jewitt et al. 2000; Nakamura \& Yoshida 2008).

Jupiter Trojans are thought to have been captured from a much larger population of small bodies (planetesimals) that existed in the planetary region $(\sim 5-30 \mathrm{AU})$ when the giant planets formed. Previous theories suggested that the Trojans were captured during the early stages of Jupiter's growth (Marzari \& Scholl 1998; Fleming \& Hamilton 2000), by collisions (Shoemaker et al. 1989), effects of nebular gas (Yoder 1979; Peale 1993; Kary \& Lissauer 1995; Kortenkamp \& Hamilton 2001), etc. These theories imply that the inclination distribution of the Trojans should be relatively narrow with most orbits having $i<10^{\circ}$ (Marzari et al. 2002). By contrast, observations show a wide inclination distribution of Jupiter Trojans with inclinations up to $\simeq 35^{\circ}$. Attempts to explain the large inclinations of Trojans by exciting orbits after capture have been unsuccessful, because passing secular resonances and other dynamical effects (e.g., Gomes 1998; Petit et al. 1999; Marzari \& Scholl 2000) are not strong enough.

Morbidelli et al. (2005, hereafter M05) proposed that Jupiter Trojans were trapped in orbits at $L_{4}$ and $L_{5}$ by chaotic capture. Chaotic capture occurs when Jupiter and Saturn pass, during their orbital migration, near the mutual 2:1 mean motion resonance (MMR), where the period ratio $P_{\text {Sat }} / P_{\text {Jup }}=2$ (today this ratio is 2.49). The angle $\lambda_{\text {Jup }}-2 \lambda_{\text {Sat }}-\varpi$, where $\varpi$ is the perihelion longitude of either Jupiter or Saturn, can then resonate with $\Delta \lambda$, creating widespread chaos around $L_{4}$ and $L_{5}$. Small bodies scattered by planets into the neighborhood of Jupiter's orbit can chaotically wander near $L_{4}$ and $L_{5}$, where they are permanently trapped once $P_{\mathrm{Sat}} / P_{\mathrm{Jup}}$ moves away from 2 .

A natural consequence of chaotic capture is that orbits fill all available space characterized by long-term stability. In M05, the planetesimals dynamically evolving from the transplanetary disk scatter off of the giant planets, acquire high-inclination orbits, and remain on these orbits after capture. This creates a wide inclination distribution of captured bodies, and resolves the long-standing conflict between previous formation theories and observations discussed above.

M05 placed chaotic capture in the context of the original Nice model (hereafter ONM; Tsiganis et al. 2005; Gomes et al. 2005), where migration of Jupiter and Saturn past their mutual 2:1 MMR is thought to trigger an instability during which Uranus and Neptune are scattered into the outer planetesimal disk. Their orbits subsequently stabilize and circularize near 20 and $30 \mathrm{AU}$ by dynamical friction (Binney \& Tremaine 1987). There are many things to like about the Nice model, but that does not mean it is correct. It needs to be continually tested against all possible constraints. Indeed, several inconsistencies of the ONM have been already pointed out leading to the model's revisions (Morbidelli et al. 2007; Levison et al. 2011).

It is now thought that Jupiter and Saturn have not smoothly migrated over the 2:1 MMR. Instead, $P_{\text {Sat }} / P_{\text {Jup }}$ probably "jumped" from $<2$ to $>2.3$ when Jupiter (and Saturn) scattered off of the ice giants (Uranus, Neptune, or a similar-mass planet). This model, known as the jumping-Jupiter model, is 
required to explain the secular architecture of the outer planets, orbital distribution of asteroids, and dynamical survival of the terrestrial planets (Morbidelli et al. 2009a, 2010; Brasser et al. 2009; Minton \& Malhotra 2009; Walsh \& Morbidelli 2011; Agnor \& Lin 2012). Moreover, encounters of Jupiter with one of the ice giants are required for capture of irregular satellites around Jupiter (Nesvorný et al. 2007).

M05's chaotic capture does not work in the jumping-Jupiter model, because the resonances discussed in M05 do not occur, and alternative capture models have not been investigated so far. Here, we test how Jupiter Trojans can be captured in the jumping-Jupiter model. We find that the Trojans are most likely captured immediately after a close encounter of Jupiter with an ice giant. As a result of the encounter, $a_{\text {Jup }}$ changes, sometimes by as much as $\sim 0.2 \mathrm{AU}$ in a single jump. This radially displaces Jupiter's $L_{4}$ and $L_{5}$, releases the existing Trojans, and can lead to capture of new bodies that happen to have semimajor axes similar to $a_{\text {Jup }}$ when the jump occurs. We call this jump capture.

\section{CAPTURE SIMULATIONS}

We take advantage of the results published in Nesvorný \& Morbidelli (2012; hereafter NM12). NM12 performed nearly $10^{4}$ numerical integrations of the early solar system's instability. The integrations started at the time when the giant planets were already fully formed and nebular gas was dispersed (presumably 3-10 Myr after the birth of the Sun; Haisch et al. 2001; Williams \& Cieza 2011). At this time, the giant planets were assumed to have orbits in mutual MMRs (that have been established during the previous stage of convergent planetary migration in the gas disk; Masset \& Snellgrove 2001; Pierens \& Nelson 2008; Pierens \& Raymond 2011). A disk of planetesimals was placed beyond the outermost ice giant (hereafter transplanetary disk). The dynamical evolution of the planets and planetesimals was then tracked, using an $N$-body integrator, through and 100 Myr past the instability.

Here, we select three cases from NM12. Their properties are illustrated in Figures 1-3. In all three cases, the solar system was assumed to have five planets initially (Jupiter, Saturn, and three ice giants). This is because NM12 showed that various constraints (such as the final orbits of outer planets, survival of the terrestrial planets, etc.) can most easily be satisfied when the system starts with five initial planets and one ice giant is ejected during the instability (Nesvorný 2011; Batygin et al. 2012). The case with four initial planets requires a massive planetesimal disk to avoid losing a planet, but the massive disk also tends to produce excessive dynamical damping and long-range residual migration of Jupiter and Saturn that violate constraints (Nesvorný 2011; Batygin et al. 2012). It is therefore difficult to obtain a plausible planet evolution starting with four planets (NM12 failed to identify any in their 2670 four-planet trials).

A shared property of the selected runs is that Jupiter and Saturn undergo a series of planetary encounters with the ejected ice giant. As a result of these encounters, the semimajor axes of Jupiter and Saturn evolve in discrete steps. While the semimajor axis can decrease or increase during one encounter, depending on the encounter geometry, the general trend is such that Jupiter moves inward, i.e., to shorter periods (by scattering ice giant outward), and Saturn moves outward, i.e., to longer periods (by scattering ice giant inward). This process leads to just the right kind of $P_{\text {Sat }} / P_{\text {Jup }}$ evolution during the instability (jumping Jupiter; see Section 1).
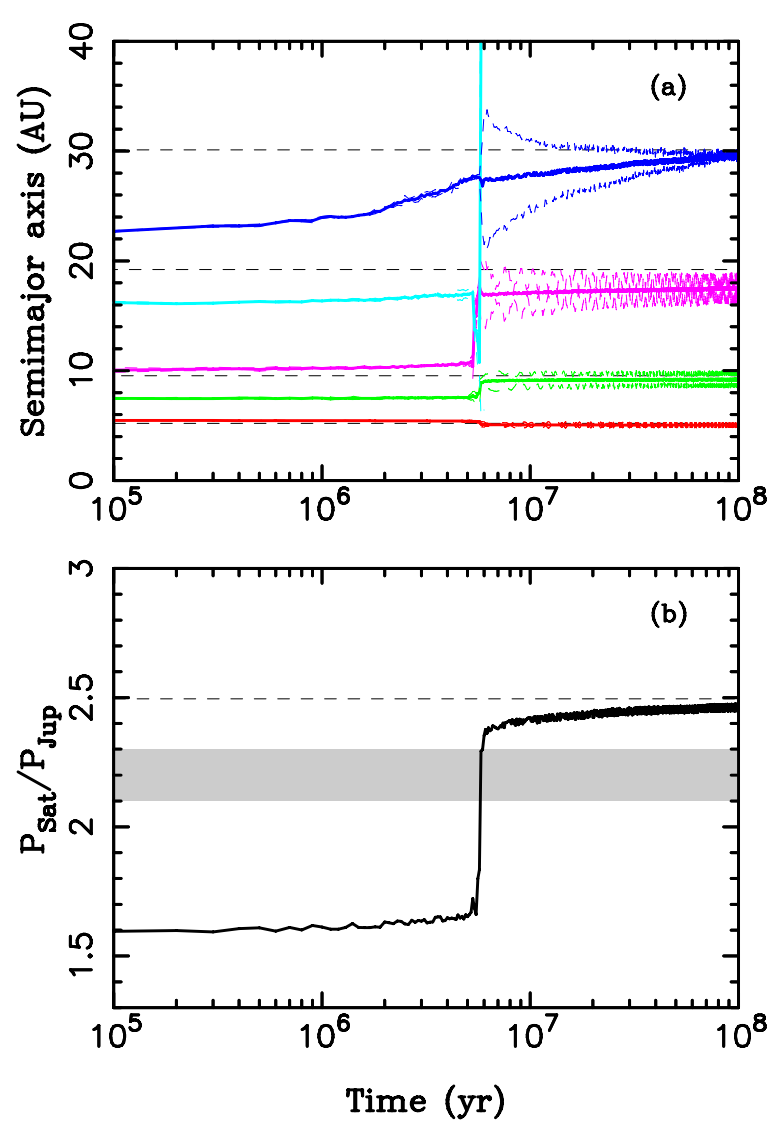

Figure 1. Orbital histories of the outer planets in Case 1. The planets were started in the $(3: 2,3: 2,2: 1,3: 2)$ resonant chain, and $M_{\text {disk }}=20 M_{\text {Earth }}$. (a) The semimajor axes (solid lines), and perihelion and aphelion distances (dashed lines) of each planet's orbit. The black dashed lines show the semimajor axes of planets in the present solar system. (b) The period ratio $P_{\text {Sat }} / P_{\text {Jup }}$. The dashed line shows $P_{\mathrm{Sat}} / P_{\mathrm{Jup}}=2.49$, corresponding to the period ratio in the present solar system. The shaded area approximately denotes the zone where the secular resonances with the terrestrial planets occur.

(A color version of this figure is available in the online journal.)

Note that while having the fifth planet is a convenient way to obtain jumping Jupiter, the fifth planet is not (in itself) important for capture of Jupiter Trojans. Instead, their capture is controlled by the evolution of Jupiter's (and Saturn's) orbit (see Section 3.1). Therefore, if the future studies will identify plausible jumping-Jupiter cases with four planets, it is expected that the capture process described here will apply to those cases as well (unless the dynamical history of Jupiter's orbit will strongly differ from the one studied here).

NM12's simulations were performed using the symplectic integrator known as SyMBA (Duncan et al. 1998). The planetesimal disk was resolved by up to 10,000 disk particles in NM12, which was sufficient for instability calculations, but will be insufficient here where the expected capture probability is $<10^{-5}$ (M05). To deal with this issue, we developed a new method that allows us to track the planetary evolution taken from the original SyMBA run, and include a very large number of disk particles whose orbits are numerically integrated by the swift_rmvs3 code, part of the Swift package (Levison \& Duncan 1994). This works as follows.

We first repeat the selected NM12 jobs using SyMBA, and record the planetary orbits at $1 \mathrm{yr}$ time intervals. Our modified version of swift_rmvs3 then reads the planetary orbits from a file, and interpolates them to any required time sub-sampling (generally $0.25 \mathrm{yr}$, which is the integration time step used 

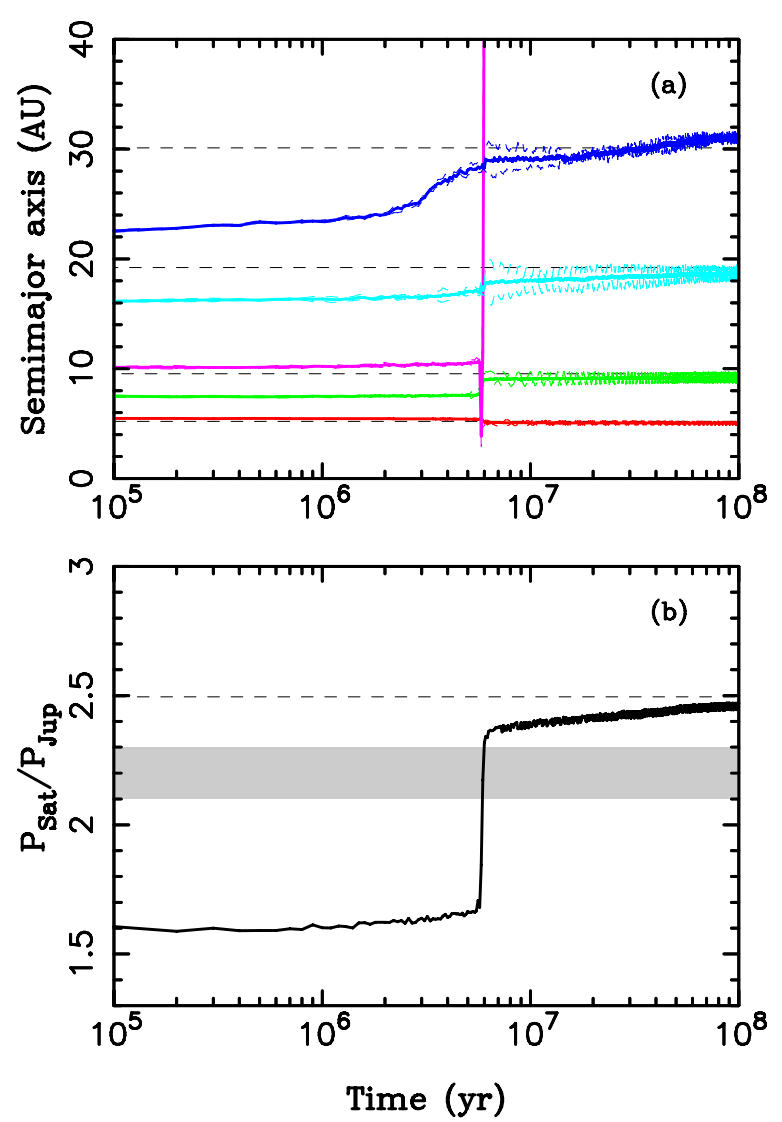

Figure 2. Orbital histories of the outer planets in Case 2. See the caption of Figure 1 for the description of orbital parameters shown here.

(A color version of this figure is available in the online journal.)

here in swift_rmvs3). The interpolation is done in Cartesian coordinates. First, the planets are forward propagated on the ideal Keplerian orbits starting from the positions and velocities recorded by SyMBA at the beginning of each 1 yr interval. Second, the SyMBA position and velocities at the end of each $1 \mathrm{yr}$ interval are propagated backward (again on the ideal Keplerian orbits). We then calculate a weighted mean of these two Keplerian trajectories for each planet so that progressively more (less) weight is given to the backward (forward) trajectory as time approaches the end of the $1 \mathrm{yr}$ interval. We verified that this interpolation method produces insignificant errors.

The swift_rmvs3 jobs were launched on different CPUs, with each CPU computing the orbital evolution of a large number of disk particles $\left(N_{\text {disk }}\right)$. The initial orbital distribution of each particle set was chosen to respect the initial distribution in the original simulation, but differed in details (e.g., the initial mean longitudes of particles were random), so that each set behaved like an independent statistical sample. This allowed us to build up good statistics. To further improve the statistics, particles were cloned upon first reaching the heliocentric distance $r<8$ AU. Particles reaching $r<8$ AU were cloned by adding $N_{\text {clo }}$ particles with orbits produced by a small (random) perturbation of the orbital velocity vector (relative magnitude $\sim 10^{-5}$ ).

We used a modest number of clones in the initial runs $\left(N_{\text {clo }}=2-4\right)$. Upon convincing ourselves that a more aggressive cloning leads to correct results (e.g., captured particles come from different clones), we used $N_{\text {disk }}=5000$ per CPU, $N_{\text {clo }}=19$, and a large number of CPUs to obtain an effective resolution with 50 million disk particles in Cases 1 and 2, and
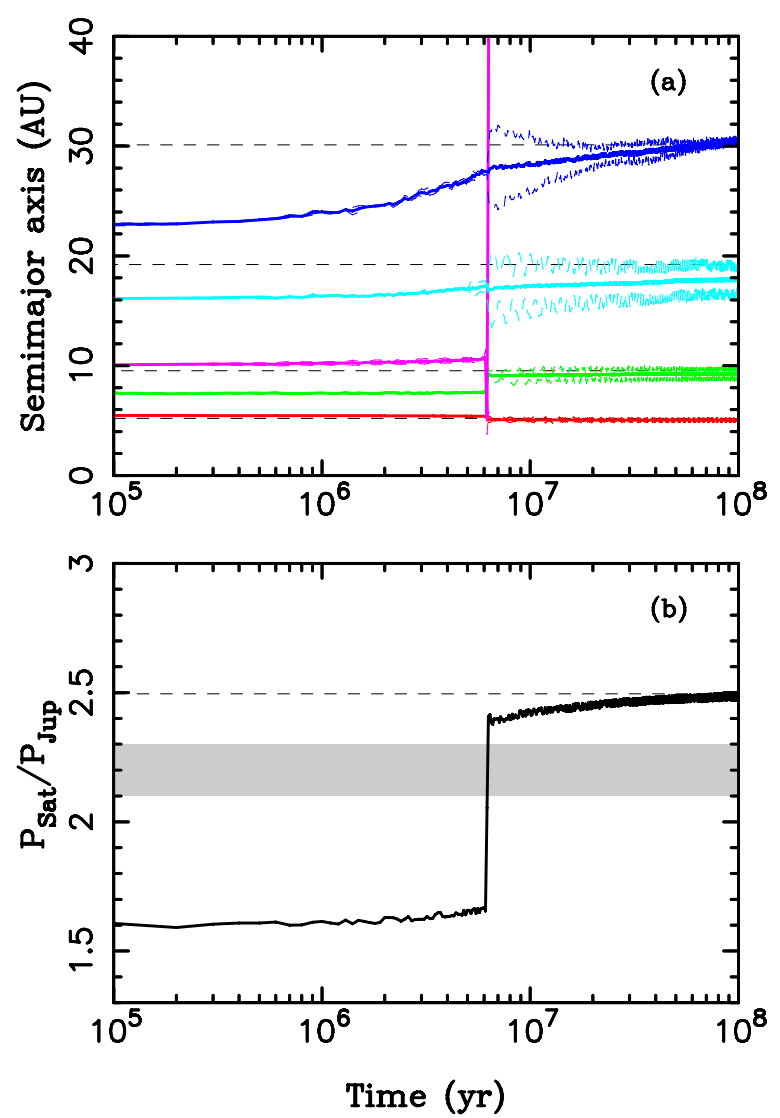

Figure 3. Orbital histories of the giant planets in Case 3. See the caption of Figure 1 for the description of orbital parameters shown here.

(A color version of this figure is available in the online journal.)

Table 1

The Statistics of Trojan Capture

\begin{tabular}{lccc}
\hline \hline & Case 1 & Case 2 & Case 3 \\
\hline$N_{\text {disk }}\left(10^{6}\right)$ & 50 & 50 & 25 \\
$N_{\text {cap }}$ & 30 & 41 & 17 \\
$P_{\text {cap }}\left(10^{-7}\right)$ & $6.0 \pm 1.1$ & $8.2 \pm 1.3$ & $6.8 \pm 1.6$ \\
$f_{45}$ & 1.3 & 0.71 & 0.55 \\
\hline
\end{tabular}

Notes. The rows are the (1) number of disk particles $\left(N_{\text {disk }}\right)$, (2) number of captured stable Trojans $\left(N_{\text {cap }}\right)$, (3) probability of capture $\left(P_{\text {cap }}\right)$, and (4) asymmetry in the number of $L_{4}$ and $L_{5}$ Trojans $\left(f_{45}=N\left(L_{4}\right) / N\left(L_{5}\right)\right)$.

25 million in Case 3 (Table 1). The whole project was concluded over a period of 8 months.

The numerical integrations described above were run from a few Myr before the instability to a few Myr after the onset of the instability (total integration timespan of $10 \mathrm{Myr}$; Phase 1). A much longer integration was difficult to achieve, because the interpolation method described above had large requirements on the computer memory (planetary positions saved at $1 \mathrm{yr}$ intervals over $10 \mathrm{Myr}$ represent gigabytes of data). Moreover, while the final planetary orbits obtained in NM12 matched the real orbits pretty well, they differed in details. For example, $P_{\text {Sat }} / P_{\text {Jup }}$ sometimes ended up being a bit lower $(\simeq 2.46)$ than in the present solar system (2.49). This difference, despite being small, would affect the long-term stability of Jupiter Trojans (e.g., Robutel \& Bodossian 2009).

We therefore continued the simulations from Phase 1 using a different method. As the planets are orbitally decoupled by 

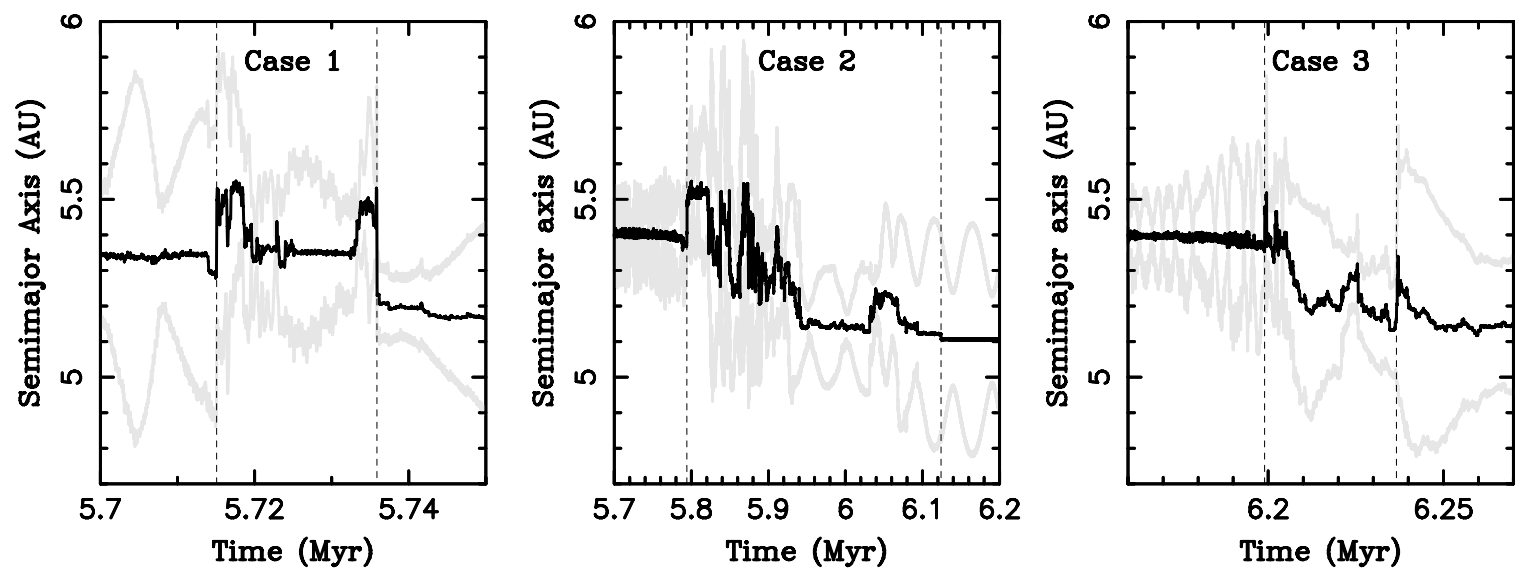

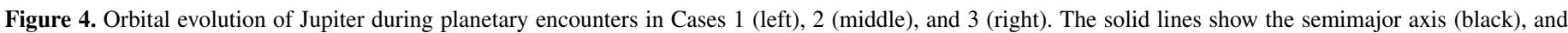
perihelion and aphelion distances (gray). The dashed vertical lines delimit the interval of Jupiter's encounters with an ice giant.

the end of Phase 1, the integrator does not need to deal with the stochastic outcomes of planetary encounters. Instead, the orbital evolution of planets during Phase 2 was governed by scattering encounters with disk particles. As a result, the planets slowly migrated toward their current semimajor axis values. This phase was followed using the swift_rmvs 3 code modified to include forces that mimic radial migration (inward for Jupiter and outward for Saturn). The migration timescale has been set to be equal to that in the original SyMBA simulations $(\simeq 30 \mathrm{Myr}$ $e$-folding timescale). The final orbits of Jupiter and Saturn were tuned so that $P_{\text {Sat }} / P_{\text {Jup }}=2.49$ in the end. Using artificial forces we will also slowly damp planetary eccentricities and inclinations (Lee \& Peale 2002), in a manner that is consistent with the original evolutions.

Phase 2 simulations were run for 100 Myr. We did not follow all disk particles during Phase 2. Instead, we identified Trojan "candidates" by selecting particles with orbits near Jupiter's $L_{4}$ and $L_{5}$ at the end of Phase 1 . This selection was very liberal in that most of the (hundreds of) selected bodies turned out not to be truly stable Trojans. The non-selected particles were discarded, which allowed us to cut down the CPU cost of Phase 2. One downside was that given that the source population of disk particles was removed, no Trojan captures could have occurred in our Phase 2 simulations. This should not be a problem, however, because the population of particles was already depleted at this stage, and planetary evolution during Phase 2 was not favorable for capture (no planetary encounters, no major resonance crossings, etc.).

To test the long-term stability of Trojans surviving at the end of Phase 2, we performed an additional numerical integration over 4 Gyr. This Phase 3 integration used the original swift_rmvs3 code and 0.25 -yr timestep. We found that the long-term stability requirements shaved off about $50 \%$ of Trojans from the population that survived at the end of Phase 2. The removed particles typically had $D>60^{\circ}$, large $e$ and/or large $i$. This result is consistent with the expected stability of Jupiter Trojans (Levison et al. 1997; Nesvorný \& Dones 2002; Robutel \& Gabern 2006).

\section{RESULTS}

Here, we discuss the results of the numerical integrations described in the previous section. The mechanism of jump capture is illustrated in Section 3.1. We then examine the orbital distribution of captured Trojans and compare it with observations (Section 3.2). The efficiency of jump capture and its implications for the size distribution of planetesimals in the transplanetary disk are discussed in Section 3.3. In Section 3.4, we point the possible source of asymmetry between the populations of $L_{4}$ and $L_{5}$ Trojans.

\subsection{Capture Process}

While the global evolution of planets was similar in the three selected cases (Figures 1-3), the detailed behavior of Jupiter's orbit differed from case to case (Figure 4). This difference can be important for Trojan capture and is why different cases were selected in the first place. In Case 1, several very close encounters between Jupiter and an ice giant occurred near the end of the scattering phase $(t=5.736 \mathrm{Myr})$ producing a cumulative change of $a_{\text {Jup }}$ from 5.53 to 5.2 AU. In Case 2, the scattering phase of Jupiter lasted over $300 \mathrm{kyr}$ with many close encounters contributing to changes of $a_{\text {Jup }}$. In contrast, Case 3 showed a relatively poor history of Jupiter's encounters lasting 40 kyr only.

By analyzing these different cases we found that capture of most Jupiter Trojans generally occurred during the stage of Jupiter's encounters. In Case 1, for example, roughly $70 \%$ of captured Trojans that survived to the end of Phase 3 (hereafter the stable Trojans) started librating around $L_{4}$ or $L_{5}$ at $t=5.715-5.736 \mathrm{Myr}$ after the start of Phase 1 . This clearly coincides in time with the period of Jupiter's encounters with the ice giant (see Case 1 in Figure 4). By analyzing the capture histories in detail we found that $\simeq 50 \%$ of stable Trojans in Case 1 were captured during the closest encounter between Jupiter and the ice giant at $t=5.735869 \mathrm{Myr}$, when $a_{\text {Jup }}$ jumped from 5.53 to $5.3 \mathrm{AU}$ (the ice giant was scattered to a very eccentric orbit as a result of this encounter).

The particles captured at $t=5.735869$ Myr had special orbits just before the encounter $\left(a \simeq 5.3 \mathrm{AU}\right.$, low $e$ and $i \lesssim 30^{\circ}$ ). They were scattered to these orbits by previous encounters with planets (Figure 5). They were subsequently captured in librating trajectories around $L_{4}$ or $L_{5}$ when the Lagrange points got displaced to $\simeq 5.3 \mathrm{AU}$ as a result of Jupiter's semimajor axis jump. This is a clear example of jump capture. In addition, roughly $10 \%$ of the stable Trojans were jump captured during the previous encounter at $t=5.715 \mathrm{Myr}$ when $a_{\text {Jup }}$ increased (Figure 5). They survived on librating trajectories during the closest encounter at $t=5.735869$ Myr only because they had the right libration phase during the encounter (so that $a \simeq 5.3 \mathrm{AU}$ ). 

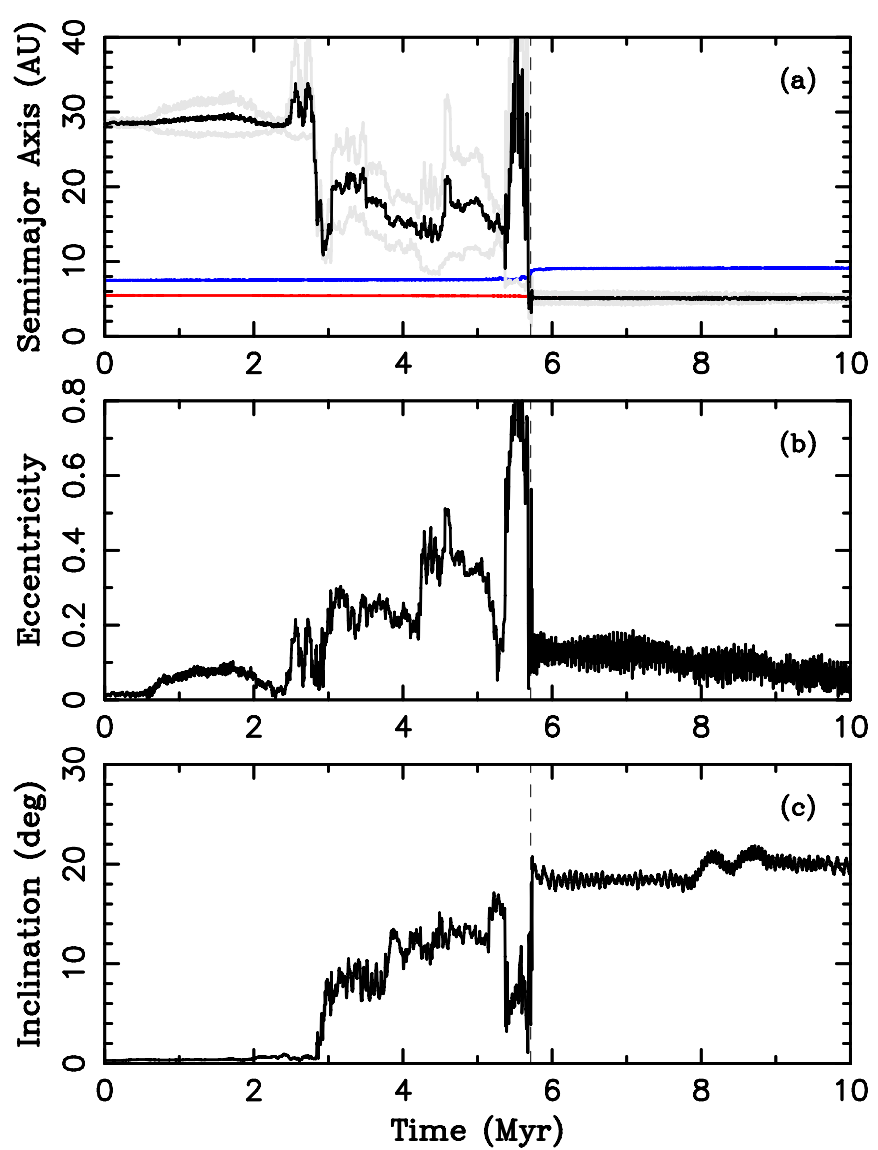

Figure 5. Orbital evolution of a disk particle that was captured as a stable Trojan in Case 1: (a) semimajor axis (black line), and perihelion and aphelion distances (gray lines) of the particle, (b) eccentricity, and (c) inclination. Jupiter's and Saturn's semimajor axes are shown in (a) by red and blue lines, respectively. The particle orbit remained near its starting location in the transplanetary disk for up to $t=3.5 \mathrm{Myr}$ after the start of the integration. The changes of $e$ and $i$ were minor during this stage. Then, at $t=3.5 \mathrm{Myr}$, the particle was scattered by Neptune, evolved inward, and a series of subsequent encounters with ice giants raised orbit's $e$ and $i$ to moderate values. At $t=5.4 \mathrm{Myr}$, particle's eccentricity evolved to very high values $(e=0.8)$ by encounters with Saturn. Finally, shortly before $t=5.735869 \mathrm{Myr}$, when the closest encounter of Jupiter with an ice giant occurred (vertical dashed line), the particle was scattered by Jupiter. This changed its orbit in just the right way for capture to be possible at $t=5.735869 \mathrm{Myr}$ (i.e., $a \simeq 5.3 \mathrm{AU}$ and small $e$ prior to capture). The large inclination of captured orbit was established by several scattering encounters with Jupiter shortly before capture.

(A color version of this figure is available in the online journal.)

Out of the remaining $\sim 40 \%$ of stable Case 1 Trojans roughly $20 \%$ were captured after the stage of planetary encounters was over $(t \gtrsim 5.8 \mathrm{Myr})$. Chaotic capture related to sweeping over weaker resonances, such as the 7:3, 12:5, and 17:7 MMRs, was responsible for these cases. The other $\simeq 20 \%$ showed a complicated evolution that was difficult to classify. These cases probably correspond to jump capture during smaller jumps of $a_{\text {Jup }}$, to chaotic capture during the irregular evolution of $a_{\text {Jup }}$, or to the combination of both. The capture statistics in Cases 2 and 3 were broadly similar: $55 \%$ of clear jump captures, $5 \%$ of clear chaotic captures, and $40 \%$ unclear in Case 2; and $50 \%$ of jump capture, $20 \%$ of chaotic capture, and $30 \%$ unclear in Case 3.

\subsection{Final Orbits}

Figure 6 shows an example of the orbital history of a stable Trojan after its capture. The changes of the libration amplitude seen in panel (a) are related to sweeping resonances (Robutel $\&$ Bodossian 2009). The libration amplitude stabilized at $t>$ $10 \mathrm{Myr}$ as planetary migration slowed down, and the system did not encounter any important resonances when $P_{\text {Sat }} / P_{\text {Jup }}$ approached 2.49 . While the eccentricity can still significantly change after capture (panel b), the inclination of captured orbits typically remained nearly constant (panel d). This shows that the inclination distribution of Jupiter Trojans is closely related to that of planetesimals near 5 AU during the scattering phase. It is wide mainly because of scattering encounters of planetesimals with Jupiter and Saturn. ${ }^{4}$

The orbital distribution of stable Trojans produced in our simulations very closely matches observations (Figures 7 and 8). The distribution extends down to very small libration amplitudes, small eccentricities, and small inclinations. These orbits are generally the most difficult to populate in any capture model. The inclination distribution of captured objects is wide, extending up to $i \simeq 30^{\circ}$, just as needed.

To carefully compare the inclination distribution obtained in our model with observations, we should ideally need to account for the detection efficiency of objects with different inclinations. This is because most surveys look near the ecliptic and tend to have lower detection efficiencies for orbits with larger inclinations (e.g., Jewitt et al. 2000). We use a magnitude cutoff to avoid this problem. According to Szabó et al. (2007), the Trojan population should be (nearly) complete up to $H \simeq 12$. Figure 8(c) shows an excellent agreement between our model inclination distribution and the one obtained with $H<12$.

Interestingly, despite the very different histories of $a_{\text {Jup }}$ in Cases 1, 2, and 3, discussed in Section 3.1, the orbital distributions of stable Trojans that were obtained in these cases are similar (Figure 7). This indicates that jump capture is a robust capture mechanism that is expected to produce the correct orbital properties of Jupiter Trojans for a wide range of jumping-Jupiter evolutions.

We applied the Kolmogorov-Smirnov (K-S) test to the orbital distributions in Figure 8 (Press et al. 1992). The K-S test is a statistical measure indicating whether two sets of data (the computed and observed orbits of Trojans in our case) are drawn from two different distribution functions, or whether they are consistent with a single distribution function. The K-S test gives $16 \%, 68 \%$, and $63 \%$ probability that the computed and measured (with cutoff $H<12$ ) distributions of $D, e$, and $i$ are statistically the same, respectively.

The lower K-S probability for $D$ is caused by a modest shortage of model orbits with $D \lesssim 20^{\circ}$. This, however, varies from case to case. In Case 1 , the $\mathrm{K}-\mathrm{S}$ probability for $D$ is $60 \%$, while it is only $3 \%$ in Case 2 . The larger probability in Case 1 may be related to the fact that most bodies were captured in Case 1 during a single large jump of $a_{\text {Jup }}$ (Section 3.1). Such a clean jump can more easily produce $D<20^{\circ}$. Conversely, in Case 2, a more continuous evolution of $a_{\text {Jup }}$ may not allow enough bodies to evolve to $D<20^{\circ}$. These conclusions will need to be checked with better statistics.

\footnotetext{
4 The wide inclination distribution of Jupiter Trojans is therefore unrelated to and cannot be used to constrain the inclination distribution of planetesimals in the original transplanetary disk. In contrast, Neptune Trojans suffered smaller inclination perturbations prior to their capture and can be used to this end (e.g., Nesvorný \& Vokrouhlický 2009).

5 A two-dimensional K-S test applied to the $e-D$ and $i-D$ distributions obtained in Case 1 gives $25 \%$ and $50 \%$ probabilities, respectively, that the computed and observed distributions are the same.
} 

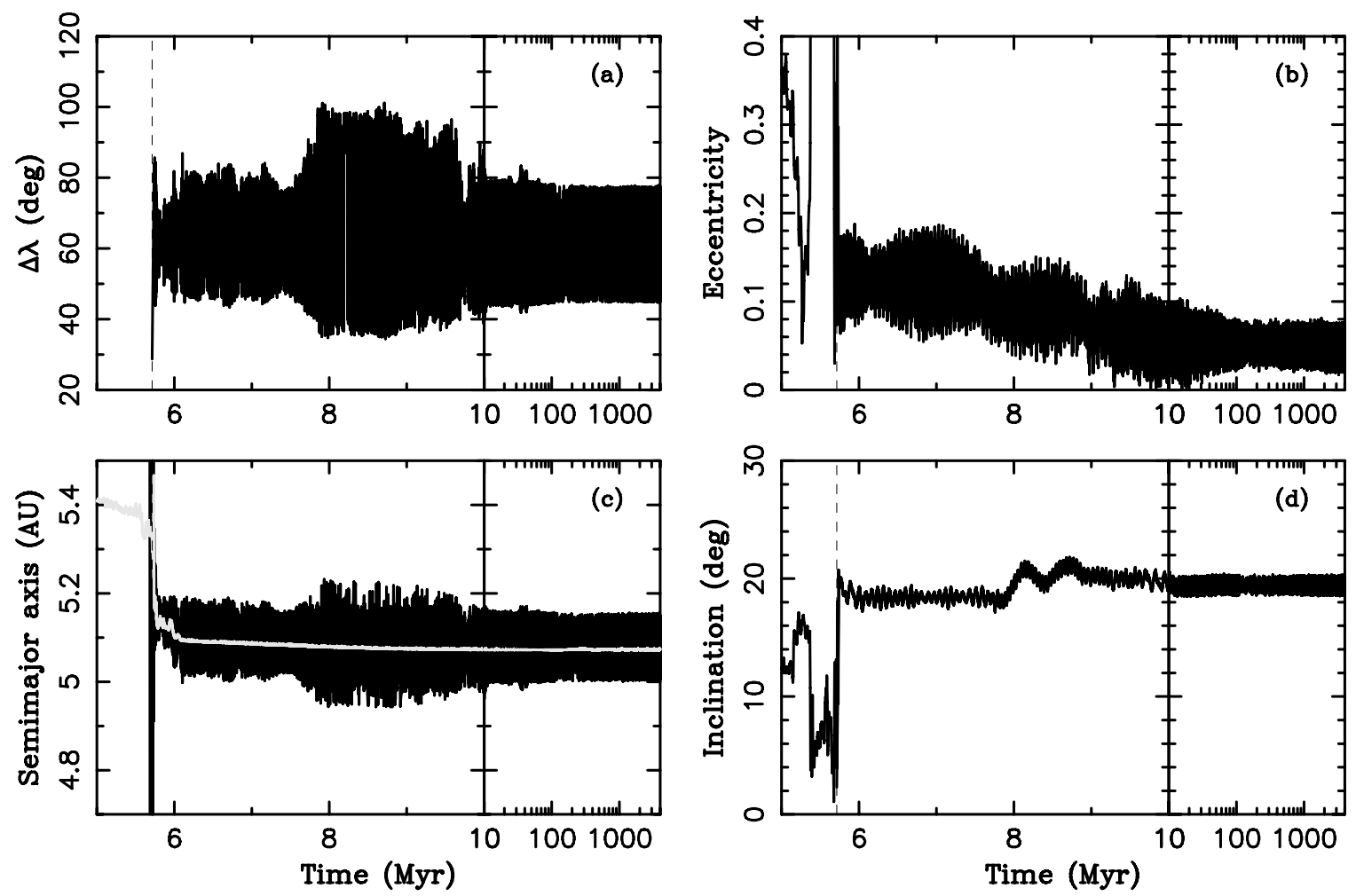

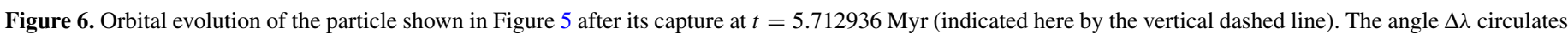
before capture and is not shown in panel (a) for $t<5.712936$ Myr. The bright gray line in panel (c) is the semimajor axis of Jupiter.

\subsection{Capture Efficiency}

We identified 30 stable Trojans in Case 1 (out of $N_{\text {disk }}=$ $5 \times 10^{7}$ disk particles), 41 in Case $2\left(N_{\text {disk }}=5 \times 10^{7}\right)$, and 17 in Case $3\left(N_{\text {disk }}=2.5 \times 10^{7}\right)$. This corresponds to the mean weighted capture efficiency of $P=(7.0 \pm 0.7) \times 10^{-7}$ for each particle in the original planetesimal disk (Table 1), where the formal $1 \sigma$ error was computed assuming the normal distribution. ${ }^{6}$ As there are 25 known Trojans with $H<9$ (this sample is complete), this indicates that the planetesimal disk contained $\sim 25 /\left(7 \times 10^{-7}\right)=3.6 \times 10^{7}$ planetesimals with $H<9$ (corresponding to diameter $D=80 \mathrm{~km}$ for a $7 \%$ albedo; Grav et al. 2012).

This is encouraging because it favorably compares with estimates obtained by other means (e.g., Nesvorný et al. 2007; Levison et al. 2008; Charnoz et al. 2009; Morbidelli et al. 2009b). For example, Charnoz et al. (2009) suggested, using the crater record on Saturn's moon Iapetus (surveyed by the Cassini spacecraft), that the planetesimal disk contained $\sim 10^{7}$ planetesimals with $D>80 \mathrm{~km}$. Morbidelli et al. (2009b), using a synthesis of constraints (mainly from the Kuiper Belt), proposed that the disk contained $\sim 10^{8}$ planetesimals with $H<9$.

The stable Trojans captured in our simulations sample the full radial extent of the transplanetary disk up to $\sim 30 \mathrm{AU}$ (at least we were not able to detect any preferential sampling based on our statistics). This shows that the size frequency distribution (SFD) of Trojans should be representative for the SFD in the

\footnotetext{
6 The capture efficiency is given here for each particle in the original transplanetary disk. M05 instead reported, quoting, "capture efficiencies per one particle cycled through the system as the planets migrate through unstable Trojan configurations." M05 found that this corresponds to $3.4 M_{\text {Earth }}$ in the reference simulation of Tsiganis et al. (2005). As Tsiganis et al. used $M_{\text {disk }} \sim 35 M_{\text {Earth }}$, the capture efficiencies reported in M05, $1.8 \times 10^{-5}$ to $2.4 \times 10^{-6}$, should be divided by $\sim 10$ to compare them to our values.
}

whole transplanetary disk (at least) up to $\sim 30$ AU (Morbidelli et al. 2009b).

M05 estimated that the present mass of the Trojan population

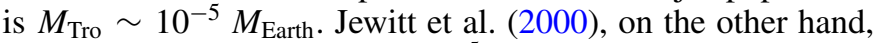
suggested that $M_{\text {Tro }} \sim 9 \times 10^{-5} M_{\text {Earth. Using bulk density }}$ $\rho=1 \mathrm{~g} \mathrm{~cm}^{-3}$ (e.g., Marchis et al. 2006) instead of Jewitt's $\rho=2 \mathrm{~g} \mathrm{~cm}^{-3}$, and updated albedo (7\% from Grav et al. 2012 instead of Jewitt's $4 \%$ ), we find that $M_{\text {Tro }} \sim 2 \times 10^{-5} M_{\text {Earth }}$. With $M_{\text {Tro }} \sim(1-2) \times 10^{-5} M_{\text {Earth }}$, we can therefore estimate that the planetesimal disk mass was $\sim(1-2) \times 10^{-5} /\left(7 \times 10^{-7}\right)=$ 14-28 $M_{\text {Earth }}$. This is consistent with $M_{\text {disk }}=20 M_{\text {Earth }}$ used in NM12.

\section{4. $L_{4} / L_{5}$ Asymmetry}

The difference in the number of leading and trailing Trojans is a long-standing problem in planetary science (see Marzari et al. 2002 for a review). This is because all capture mechanisms proposed so far, including chaotic capture of M05, are expected to produce symmetric distributions with the numbers of bodies in each swarm, $N\left(L_{4}\right)$ and $N\left(L_{5}\right)$, being nearly the same (up to statistical fluctuations). Planetary migration is also not expected to change $f_{45}=N\left(L_{4}\right) / N\left(L_{5}\right)$, nor is the long-term stability. ${ }^{7}$ In addition, the existing asymmetry is not simply due to different collisional evolution of the $L_{4}$ and $L_{5}$ Trojan swarms, because it persists even if known collisional families at $L_{4}$ are removed (O’Brien \& Morbidelli 2008).

Various research groups published estimates of $f_{45}$ that apply to different limiting magnitudes/sizes. Szabó et al. (2007)

\footnotetext{
7 Gomes (1998) claimed that planetary migration can change $f_{45}$, but these changes were probably related to the choice of the initial orbits in Gomes (1998) rather than to an asymmetry of the effects of planetary migration itself (e.g., O'Brien 2012).
} 

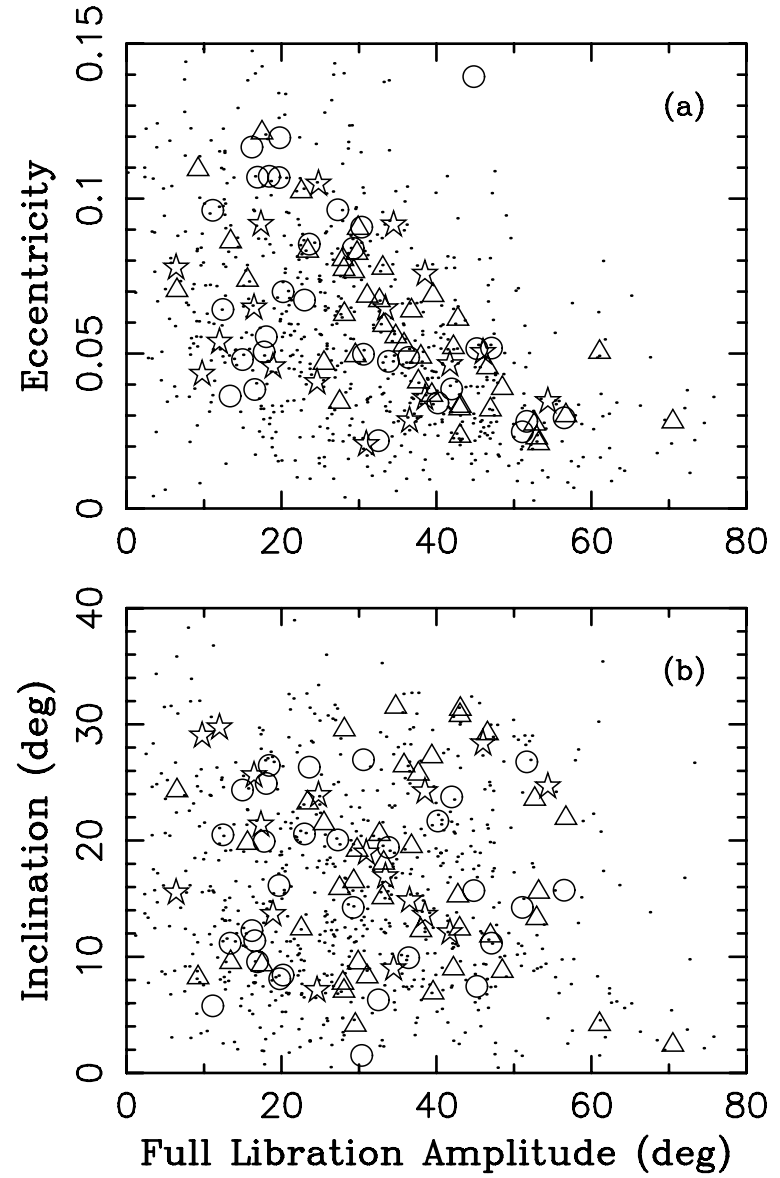

Figure 7. Orbits of stable Trojans obtained in Cases 1 (circles), 2 (triangles), and 3 (stars). The full libration amplitude $D$ corresponds to the angular distance between extremes of $\lambda-\lambda_{\text {Jup }}$ during libration. The black dots show the orbital distribution of real Trojans. The proper orbital elements shown here were computed by the method described in Brož \& Rozehnal (2011). We found no significant difference between the orbital distributions of $L_{4}$ and $L_{5}$ Trojans, and clumped these distributions together.

estimated from the Sloan Digital Sky Survey that $f_{45}=$ $1.6 \pm 0.1$. Survey with the Subaru telescope gave $f_{45} \simeq 1.8$ (Nakamura \& Yoshida 2008). Estimates from the Wide-field Infrared Survey Explorer gave $f_{45}=1.4 \pm 0.2$ (Grav et al.

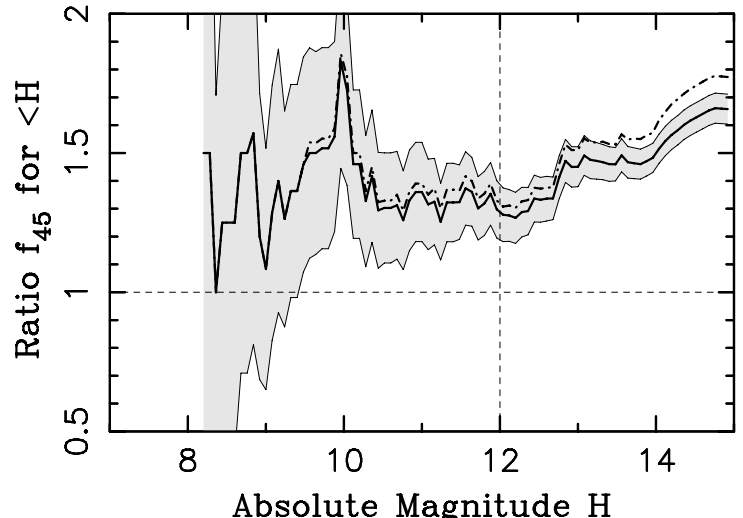

Figure 9. Asymmetry between the populations of $L_{4}$ and $L_{5}$ Trojans. The solid line shows $f_{45}=N\left(L_{4}\right) / N\left(L_{5}\right)$ for the known Trojans as a function of absolute magnitude $H$. The Eurybates family, as identified by Brož \& Rozehnal (2011), was removed from $N\left(L_{4}\right)$ (the dash-dotted line shows $f_{45}$ with the Eurybates family included in $N\left(L_{4}\right)$ ). The gray region denotes our $1 \sigma$ statistical error estimate of $f_{45}$.

2011) and $f_{45} \simeq 1.34$ for diameters $D>50 \mathrm{~km}$ (Grav et al. 2012). Therefore, $f_{45}=1.2-1.8$ according to these works.

Figure 9 shows $f_{45}$ of the known Jupiter Trojans as a function of $H$ (data from the Minor Planet Center). The ratio is wiggly for $H \lesssim 10$, because only a very few bright Trojans exist. The statistics for $H<9$ is apparently not large enough to rule out $f_{45}=1$ with confidence. At the faint end, on the other hand, the sample is incomplete, and $f_{45}$ can be influenced by a few large collisions that generated a lot of small debris (e.g., Brož \& Rozehnal 2011).

To assess the significance of the asymmetry we find it best to use the population with $H<12$ (complete sample according to Szabó et al. 2007). The number of the known $L_{4}$ Trojans with $H<12$ is 361, if 9 known Eurybates family members with $H<12$ are removed (Brož \& Rozehnal 2011). For comparison, there are $279 L_{4}$ Trojans with $H<12$. This indicates that $f_{45}=1.3 \pm 0.1$, where the formal error was computed as $f_{45}\left[1 / N\left(L_{4}\right)+1 / N\left(L_{5}\right)\right]^{0.5}$. The asymmetry is therefore significant at $\simeq 3 \sigma$ for $H<12$.

The jump capture discussed in Section 3.1 is potentially capable of producing an asymmetry. For example, the asymmetry can arise shortly after planetesimals are jump captured, while

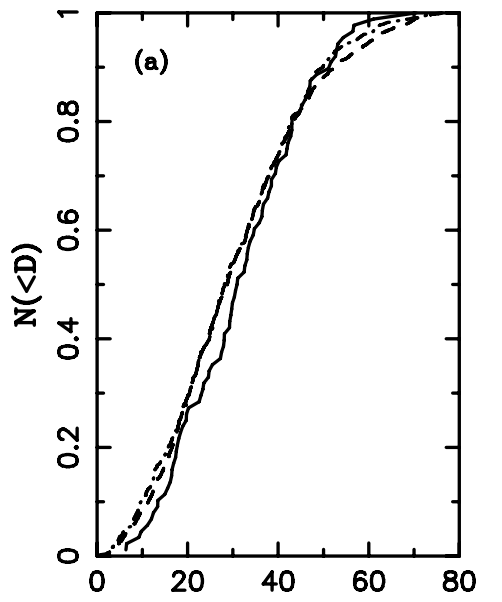

Full Libration Amplitude D (deg)
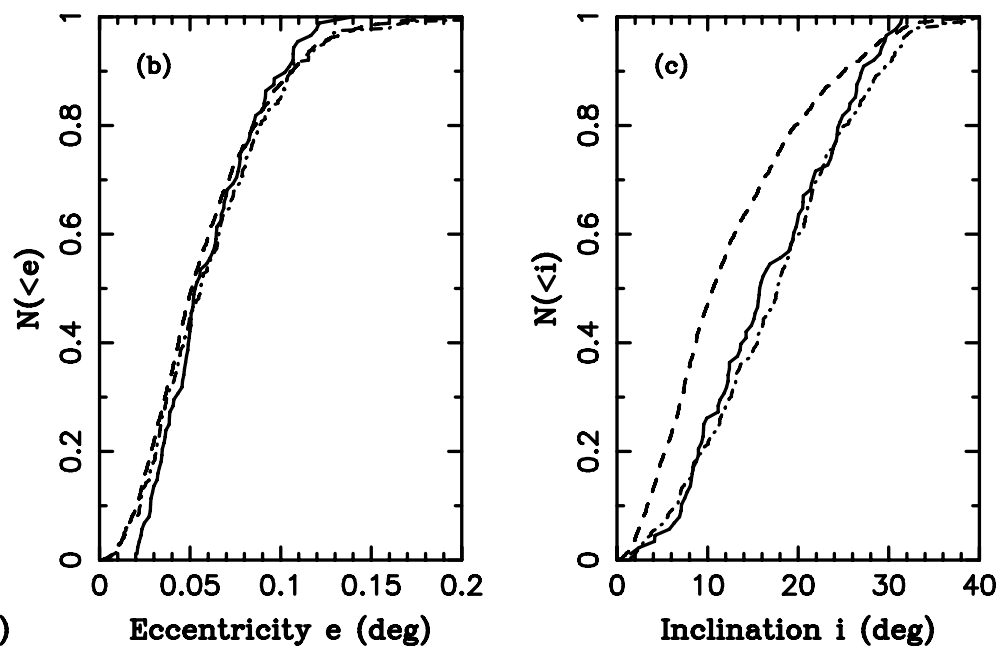

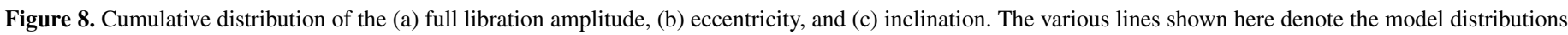
(solid), known Trojans (dashed), and known Trojans with absolute magnitude $H<12$ (dot-dashed). According to Szabó et al. (2007), the population of known Trojans with $H<12$ should be nearly complete. The difference between the dashed and dot-dashed lines in panel (c) is related to the incompleteness of the faint Trojans with high orbital inclinations. 
the ice giant still remains on a Jupiter-crossing orbit. During this time, the ice giant can traverse one of the Lagrange swarms and scatter captured bodies, causing the local population to drop. If so, the observed $f_{45}>1$ would indicate that the ice giant traversed the $L_{5}$ cloud shortly after the bulk of the Trojan population was captured. Note that this source of asymmetry does not apply to chaotic capture described in M05, because the orbits of ice giants do not reach down to Jupiter's orbit in M05.

From our simulations, we get $f_{45}=1.3 \pm 0.5$ in Case 1 , $f_{54} \equiv 1 / f_{45}=1.4 \pm 0.4$ in Case 2 , and $f_{54}=1.8 \pm 0.9$ in Case 3 . While all three cases therefore show a formal asymmetry, the statistics is not good enough to rule out $f_{45}=1$ at more than $1 \sigma$. It is therefore possible that we are just seeing statistical fluctuations of a small sample. Unfortunately, increasing the statistics to $3 \sigma$ with the method described in Section 2 is not computationally feasible at this time, because we would need to increase the number of disk particles by a factor of $\sim 10$. We leave this issue for a future work.

\section{CONCLUSIONS}

Here, we discussed a new model for capture of Jupiter Trojans. The jump capture, as we call it, occurs when Jupiter undergoes a series of scattering encounters with an ice giant, and $a_{\text {Jup }}$ evolves through a number of discrete steps as a result of these encounters. We performed numerical integrations of jump capture, where orbits of 125 million disk planetesimals were tracked over the period of discrete changes of $a_{\text {Jup }}$. The captured bodies were followed from the time of their capture, presumably some 4 Gyr ago, to the present time. The number and orbits of the surviving bodies were compared with observations of Jupiter Trojans. Our results are summarized as follows.

1. We found that the efficiency of jump capture is $(6-8) \times 10^{-7}$ for each particle in the original transplanetary disk. This, and the number of known Trojans with $H<9$, imply that the planetesimal disk contained $(3-4) \times 10^{7}$ bodies with $H<9$ (corresponding to diameter $D=80 \mathrm{~km}$ for a $7 \%$ albedo). The inferred mass of the planetesimal disk is $M_{\text {disk }}=14-28 M_{\text {Earth }}$.

2. The orbital distribution of stable Trojans obtained in our simulations provides a good match to the observed distribution, including orbits with small libration amplitudes, small eccentricities, and small/large inclinations. The present wide inclination distribution of Jupiter Trojans reflects the distribution of planetesimals near 5 AU during the planetary instability.

3. The jump capture is potentially capable of explaining the observed asymmetry of Jupiter Trojans $\left(N\left(L_{4}\right) / N\left(L_{5}\right)=\right.$ $1.3 \pm 0.1$ for the complete sample with $H<12)$. The asymmetry can be related to (a few significant) late passages of an ice giant near $L_{5}$ that presumably depleted the $L_{5}$ population. Future modeling work will need to improve the capture statistics and test this possibility at a larger statistical confidence that it was done here.

In a broader context, the work presented here provides support for the jumping-Jupiter model (Morbidelli et al. 2009a, 2010; Brasser et al. 2009), and shows a good consistency of the planetary-instability simulations published in NM12.

This work was supported by NASA's Outer Planet Research programs. Alessandro Morbidelli thanks Germany's Helmholtz Alliance for providing support through its Planetary Evolution and Life program. David Nesvorný thanks the Observatoire de la Côte d'Azur for hospitality during his sabbatical year in Nice. The work of David Vokrouhlický was partly supported by the Czech Grant Agency (grant 205/08/0064). We thank M. Brož for helping us with the computation of the proper elements.

\section{REFERENCES}

Agnor, C. B., \& Lin, D. N. C. 2012, ApJ, 745, 143

Batygin, K., Brown, M. E., \& Betts, H. 2012, ApJL, 744, L3

Binney, J., \& Tremaine, S. 1987, Galactic Dynamics (Princeton, NJ: Princeton Univ. Press)

Brasser, R., Morbidelli, A., Gomes, R., Tsiganis, K., \& Levison, H. F. 2009, A\&A, 507, 1053

Brož, M., \& Rozehnal, J. 2011, MNRAS, 414, 565

Charnoz, S., Morbidelli, A., Dones, L., \& Salmon, J. 2009, Icar, 199, 413

Duncan, M. J., Levison, H. F., \& Lee, M. H. 1998, AJ, 116, 2067

Fleming, H. J., \& Hamilton, D. P. 2000, Icar, 148, 479

Gomes, R. S. 1998, AJ, 116, 2590

Gomes, R., Levison, H. F., Tsiganis, K., \& Morbidelli, A. 2005, Natur, 435, 466

Grav, T., Mainzer, A. K., Bauer, J., et al. 2011, ApJ, 742, 40

Grav, T., Mainzer, A. K., Bauer, J. M., Masiero, J. R., \& Nugent, C. R. 2012, ApJ, 759,49

Haisch, K. E., Jr., Lada, E. A., \& Lada, C. J. 2001, ApJL, 553, L153

Jewitt, D. C., Trujillo, C. A., \& Luu, J. X. 2000, AJ, 120, 1140

Kary, D. M., \& Lissauer, J. J. 1995, Icar, 117, 1

Kortenkamp, S. J., \& Hamilton, D. P. 2001, BAAS, 33, 1086

Lee, M. H., \& Peale, S. J. 2002, ApJ, 567, 596

Levison, H. F., \& Duncan, M. J. 1994, Icar, 108, 18

Levison, H. F., Morbidelli, A., Tsiganis, K., Nesvorný, D., \& Gomes, R. 2011, AJ, 142, 152

Levison, H. F., Morbidelli, A., Vanlaerhoven, C., Gomes, R., \& Tsiganis, K. 2008, Icar, 196, 258

Levison, H. F., Shoemaker, E. M., \& Shoemaker, C. S. 1997, Natur, 385, 42

Marchis, F., Hestroffer, D., Descamps, P., et al. 2006, Natur, 439, 565

Marzari, F., \& Scholl, H. 1998, A\&A, 339, 278

Marzari, F., \& Scholl, H. 2000, Icar, 146, 232

Marzari, F., Scholl, H., Murray, C., \& Lagerkvist, C. 2002, in Asteroids III, ed. W. F. Bottke, Jr., A. Cellino, P. Paolicchi, \& R. P. Binzel (Tucson, AZ: Univ. Arizona Press), 725

Masset, F., \& Snellgrove, M. 2001, MNRAS, 320, L55

Minton, D. A., \& Malhotra, R. 2009, Natur, 457, 1109

Morbidelli, A., Brasser, R., Gomes, R., Levison, H. F., \& Tsiganis, K. 2010, AJ, 140, 1391

Morbidelli, A., Brasser, R., Tsiganis, K., Gomes, R., \& Levison, H. F. 2009a, A\&A, 507, 1041

Morbidelli, A., Levison, H. F., Bottke, W. F., Dones, L., \& Nesvorný, D. 2009b, Icar, 202, 310

Morbidelli, A., Levison, H. F., Tsiganis, K., \& Gomes, R. 2005, Natur, 435, 462

Morbidelli, A., Tsiganis, K., Crida, A., Levison, H. F., \& Gomes, R. 2007, AJ, 134,1790

Nakamura, T., \& Yoshida, F. 2008, PASJ, 60, 293

Nesvorný, D. 2011, ApJL, 742, L22

Nesvorný, D., \& Dones, L. 2002, Icar, 160, 271

Nesvorný, D., \& Morbidelli, A. 2012, AJ, 144, 117

Nesvorný, D., \& Vokrouhlický, D. 2009, AJ, 137, 5003

Nesvorný, D., Vokrouhlický, D., \& Morbidelli, A. 2007, AJ, 133, 1962

O'Brien, D. P. 2012, AAS/Division for Planetary Sciences Meeting Abstracts, 44, 210.10

O’Brien, D. P., \& Morbidelli, A. 2008, Asteroids, Comets, Meteors (LPI Contributions 1405), 8367

Peale, S. J. 1993, Icar, 106, 308

Petit, J.-M., Morbidelli, A., \& Valsecchi, G. B. 1999, Icar, 141, 367

Pierens, A., \& Nelson, R. P. 2008, A\&A, 482, 333

Pierens, A., \& Raymond, S. N. 2011, A\&A, 533, A131

Press, W. H., Teukolsky, S. A., Vetterling, W. T., \& Flannery, B. P. 1992, Numerical Recipes in C. The Art of Scientific Computing (2nd ed.; Cambridge: Univ. Press)

Robutel, P., \& Bodossian, J. 2009, MNRAS, 399, 69

Robutel, P., \& Gabern, F. 2006, MNRAS, 372, 1463

Shoemaker, E. M., Shoemaker, C. S., \& Wolfe, R. F. 1989, Asteroids II (Tucson, AZ: Univ. Arizona Press), 487

Szabó, G. M., Ivezić, Ž., Jurić, M., \& Lupton, R. 2007, MNRAS, 377, 1393

Tsiganis, K., Gomes, R., Morbidelli, A., \& Levison, H. F. 2005, Natur, 435, 459

Walsh, K. J., \& Morbidelli, A. 2011, A\&A, 526, A126

Williams, J. P., \& Cieza, L. A. 2011, ARA\&A, 49, 67

Yoder, C. F. 1979, Icar, 40, 341 